\title{
Fast Healthcare Interoperability Resources (FHIR) in a FAIR Metadata Registry for COVID-19 Research
}

\author{
Sophie Anne Ines KLOPFENSTEIN ${ }^{\mathrm{a}, \mathrm{b}, 1}$, Carina Nina VORISEK, \\ Aliaksandra SHUTSKO ${ }^{c}$, Moritz LEHNEa, Julian SASS ${ }^{a}$, Matthias LÖBEd, \\ Carsten Oliver SCHMIDT ${ }^{\mathrm{e}}$ and Sylvia THUN $^{\mathrm{a}}$ \\ ${ }^{a}$ Core Facility Digital Medicine and Interoperability, Berlin Institute of Health at \\ Charité - Universitätsmedizin Berlin, Germany \\ ${ }^{\mathrm{b}}$ Institute of Medical Informatics, Charité - Universitätsmedizin Berlin, Germany \\ ${ }^{c} Z B M E D$ - Information Centre for Life Sciences, Germany \\ ${ }^{d}$ Institute for Medical Informatics (IMISE), University of Leipzig, Germany \\ ${ }^{e}$ Institute of Community Medicine, University Medicine Greifswald, Germany
}

\begin{abstract}
Adopting international standards within health research communities can elevate data FAIRness and widen analysis possibilities. The purpose of this study was to evaluate the mapping feasibility against $\mathrm{HL}^{\circledR}{ }^{\circledR}$ Fast Healthcare Interoperability Resources ${ }^{\circledR}$ (FHIR) ${ }^{\circledR}$ of a generic metadata schema (MDS) created for a central search hub gathering COVID-19 health research (studies, questionnaires, documents $=$ MDS resource types). Mapping results were rated by calculating the percentage of FHIR coverage. Among 86 items to map, total mapping coverage was $94 \%$ : $50(58 \%)$ of the items were available as standard resources in FHIR and 31 (36\%) could be mapped using extensions. Five items (6\%) could not be mapped to FHIR. Analyzing each MDS resource type, there was a total mapping coverage of $93 \%$ for studies and $95 \%$ for questionnaires and documents, with $61 \%$ of the MDS items available as standard resources in FHIR for studies, $57 \%$ for questionnaires and $52 \%$ for documents. Extensions in studies, questionnaires and documents were used in $32 \%, 38 \%$ and $43 \%$ of items, respectively. This work shows that FHIR can be used as a standardized format in registries for clinical, epidemiological and public health research. However, further adjustments to the initial MDS are recommended - and two additional items even needed when implementing FHIR. Developing a MDS based on the FHIR standard could be a future approach to reduce data ambiguity and foster interoperability.
\end{abstract}

Keywords. Metadata Standards, COVID-19, FAIR data, HL7 FHIR, Fast Healthcare Interoperability Resources, Syntactic Interoperability, Infrastructure

\section{Introduction}

The NFDI4Health Task Force Covid-19 (TF C19) is a project conducted by partners of the National Research Data Infrastructure for Personal Health Data (NFDI4Health). TF C19 aims to develop a FAIR (findable, accessible, interoperable and reusable) data

\footnotetext{
${ }^{1}$ Corresponding Author, Core Facility Digital Medicine and Interoperability, Berlin Institute of Health at Charité - Universitätsmedizin and Institute of Medical Informatics, Charité - Universitätsmedizin, Charitéplatz 1, 10117 Berlin, Germany; E-mail: sophie.klopfenstein@charite.de
} 
infrastructure for COVID-19 research in Germany and to foster cooperation between clinical, epidemiological and public health communities $[1,2]$. To gather information from different health data sources on COVID-19 (studies, questionnaires and documents), a metadata schema (MDS) was created and published $[3,4]$.

Fast Healthcare Interoperability Resources ${ }^{\circledR}\left(\mathrm{FHIR}^{\circledR}\right)$ is a standard introduced in 2011 by Health Level Seven International $\left(\mathrm{HL}^{\circledR}{ }^{\circledR}\right)$. It is used in health information technology and provides an information model that is composed of various distinct blocks of information, called resources. Resources intend to provide a definition of the structure and content to cover the information needs of most health information systems. Information not covered by the core resource data model can be captured by an extension mechanism allowing to store and exchange additional structured data. References are used to link resources to each other, while profiles define further rules and constraints on top of standard resources. As FHIR complies with reusability, composability, scalability, performance, usability, data fidelity and implementability principles, it is worthwhile to investigate supporting FHIR in a system [5]. FHIR is mainly used in clinical care, but there are also uses in health research [6,7] and a clinical trials registry [8]. To date there is no FHIR based common registry to gather health data and improve cooperation between clinical, epidemiological and Public Health domains.

Therefore, this paper investigates the feasibility of mapping the MDS to the FHIR standard to enable syntactic and semantic interoperability for NFDI4Health.

\section{Methods}

Items from the NFDI4Health TF COVID-19 MDS [4] were mapped for each MDS resource type (study, questionnaire, document) to the FHIR standard. The MDS contains two types of items, depending on the resource type they apply to: general and studies specific items. General MDS items were each mapped to the FHIR resources ResearchStudy, Questionnaire and DocumentReference while studies specific items were only mapped to ResearchStudy using FHIR resources of the most current version of HL7 FHIR Version Release 4 (FHIR ${ }^{\circledR}$ R4, v4.0.1) as mapping target [9]. Two mappers (SK, CV), both medical doctors with experience in FHIR, performed the MDS-to-FHIR resource mappings independently after analysis of each MDS item. Incongruities were discussed and solved within a larger mapping team (SK, CV, MLÖ, MLE, ST) resulting in a consolidated version of the mapping, followed up by a feasibility analysis. In some cases, this required further input from a FHIR expert (JS), or MDS expert (AS). Evaluation of the FHIR mapping was done by calculating the percentage of mapping in each category for each MDS resource type and across resource types. Mapping results were categorized based on previous literature as follows [10]: 1) Available as standard resource, 2) Available as extension, 3) Mapping to FHIR not possible

\section{Results}

Forty-four distinct items from the MDS were split into general $(\mathrm{n}=21)$ and studies specific items $(n=23)$ resulting in a total of eighty-six items to map [11]. Details on MDS resource types, FHIR resources and availability in FHIR can be found in Table 1. 
Table 1. NFDI4Health TF COVID-19 MDS-FHIR mapping, N (\%)

\begin{tabular}{llllll}
\hline $\begin{array}{l}\text { MDS } \\
\text { resource } \\
\text { types }\end{array}$ & FHIR resources & $\begin{array}{l}\text { Available as } \\
\text { standard } \\
\text { resource }\end{array}$ & $\begin{array}{l}\text { Available } \\
\text { as } \\
\text { extension }\end{array}$ & $\begin{array}{l}\text { Mapping to } \\
\text { FHIR not } \\
\text { possible }\end{array}$ & $\begin{array}{l}\text { MDS } \\
\text { items }\end{array}$ \\
\hline Studies & ResearchStudy & $27(61)$ & $14(32)$ & $3(7)$ & 44 \\
Questionnaires & Questionnaire & $12(57)$ & $8(38)$ & $1(5)$ & 21 \\
Documents & DocumentReference & $11(52)$ & $9(43)$ & $1(5)$ & 21 \\
& Total & $50(58)$ & $31(36)$ & $5(6)$ & 86 \\
\hline
\end{tabular}

Among all 86 mapped items, 50 (58\%) were available in FHIR as standard resources. Further 31 items (36\%) were available as extensions. Five $(6 \%)$ of the MDS items could not be mapped to FHIR. Analyzing the mapping across MDS resource types, $94 \%$ of the MDS items could be mapped either with standard FHIR resources or extensions. Mapping was possible for $93 \%$ of the MDS items for studies, and $95 \%$ for questionnaires and documents, respectively. Figure 1 illustrates the availability of MDS items in FHIR.

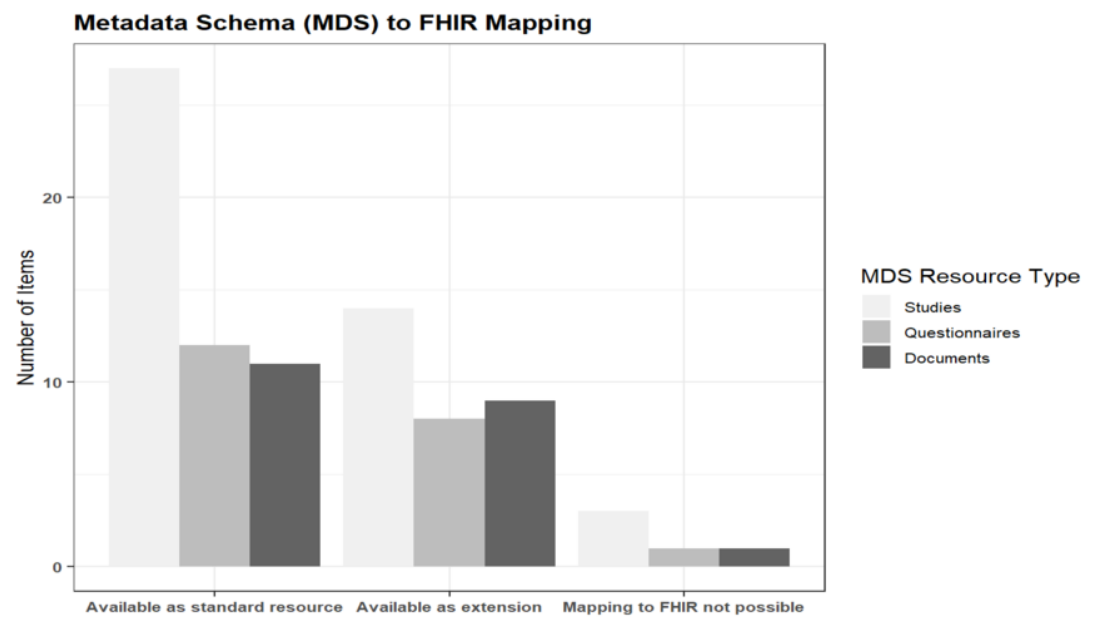

Figure 1. Number of mapped items by MDS resource type and mapping categories.

Some MDS items could not be mapped to FHIR. A mapping of the MDS items "study_status" and "study_analysis_unit" with their respective corresponding mandatory FHIR elements "ResearchStudy.status" and "Group.type" (ResearchStudy is referencing to the Group resource via ResearchStudy.enrollment) was not possible due to incompatible differences in each required value set. Building an extension would not permit to obviate the use of the value sets because of their binding strength. The MDS item "resource_type", relevant for all MDS resources, was not mapped since the selection of the MDS item "resource_type" is followed by a conditional metadata mapping to the appropriate FHIR resource.

In order to use the FHIR resources Questionnaire and DocumentReference, the following items had to be added to the MDS due to the FHIR cardinality: "Questionnaire.status", "DocumentReference.status". 


\section{Discussion}

Existing FHIR resources guarantee the coverage of common requirements but can be expanded in most of the cases using customized extensions [5]. We were able to map the majority of MDS items to FHIR either by using standard resources or custom extensions (94\%) demonstrating the flexibility of this standard and its suitability to our use case. However, FHIR resources are designed based on the $80 / 20$ rule $(20 \%$ of requirements satisfying $80 \%$ of the use cases), as well as on reusability and composability principles. With a greater number of extensions needed (36\% of the items), we might also lose the proximity to the standard and hinder the compatibility with other systems. Furthermore, analysis of the MDS items showed for example that some definitions are still ambiguous. In some cases, different concepts are covered in a unique item which might lead to complex conditional mappings. Additionally, mapping of two items was not possible because of incompatible value sets between MDS and FHIR. Adjustments of the MDS are recommended and in some cases even needed to ensure compatibility with FHIR. Generating a metadata schema based on FHIR would allow an easier integration of further standards used in various research communities while lowering the amount of FHIR extensions. The current FHIR ResearchStudy resource has a low maturity level (i.e., future changes to this resource are likely) and has a focus on clinical trials. In 2022 HL7 will release a new FHIR version. Previews of the next version show that the ResearchStudy resource will be suitable for studies beyond clinical trials [12]. Therefore, future mappings within our use case could be even more feasible. However, the exact release date is not known and main German health initiatives and projects such as the Medical Informatics Initiative Germany are using FHIR R4 [13] and compatibility is one major aspect within our the NFDI4Health initiative. Further developments should also target bridges to the OMOP data model with its focus on research databases [14].

\section{Conclusions}

The NFDI4Health TF C19 metadata schema supports a FHIR mapping and therefore can be used for different types of health resources from different research communities. A mapping of the MDS using FHIR standard resources and elements was feasible in more than half of the cases. In most of the cases where FHIR standard elements were not available, FHIR extensions were used. Five items could not be mapped and made MDS adjustments necessary. By creating a COVID-19 registry supporting FHIR, collection of structured data, findability and analysis could be leveraged in different health research communities. We plan to profile FHIR resources based on the mapping of the next metadata schema version (currently under development) and implement the created profiles. For the main project of NFDI4Health, we plan to use FHIR as a basis for a new common metadata schema, enabling syntactic interoperability and facilitating the seamless integration of further standards to ensure semantic interoperability.

\section{Acknowledgments and Competing interests}

This work was done as part of the NFDI4Health Task Force COVID-19 (www.nfdi4health.de). We gratefully acknowledge the financial support of the Deutsche 
Forschungsgemeinschaft (DFG, German Research Foundation) - Project Number 451265285, PI 345/17-1, SCHM 2744/9-1. | ST is vice chair of HL7 Deutschland e.V.

\section{References}

[1] NFDI4Health. What is NFDI4Health? [Internet]. 2020 [cited 2021 Aug 02]. Available from https://www.nfdi4health.de

[2] Wilkinson MD, Dumontier M, Aalbersberg IJ, Appleton G, Axton M, Baak A, Blomberg N, Boiten JW, da Silva Santos LB, Bourne PE, Bouwman J, Brookes AJ, Clark T, Crosas M, Dillo I, Dumon O, Edmunds S, Evelo CT, Finkers R, Gonzalez-Beltran A, Gray AJ, Groth P, Goble C, Grethe JS, Heringa J, 't Hoen PA, Hooft R, Kuhn T, Kok R, Kok J, Lusher SJ, Martone ME, Mons A, Packer AL, Persson B, Rocca-Serra P, Roos M, van Schaik R, Sansone SA, Schultes E, Sengstag T, Slater T, Strawn G, Swertz MA, Thompson M, van der Lei J, van Mulligen E, Velterop J, Waagmeester A, Wittenburg P, Wolstencroft K, Zhao J, Mons B. The FAIR Guiding Principles for scientific data management and stewardship. Sci Data. 2016 Mar 15;3:160018. doi: 10.1038/sdata.2016.18. Erratum in: Sci Data. 2019 Mar 19;6(1):6. PMID: 26978244; PMCID: PMC4792175.

[3] Schmidt CO, Fluck J, Golebiewski M, Grabenhenrich L, Hahn H, Kirsten T, Klammt S, Löbe M, Sax U, Thun S, Pigeot I; NFDI4Health Task Force Covid-19. COVID-19-Forschungsdaten leichter zugänglich machen - Aufbau einer bundesweiten Informationsinfrastruktur [Making COVID-19 research data more accessible-building a nationwide information infrastructure]. Bundesgesundheitsblatt Gesundheitsforschung Gesundheitsschutz. 2021 Jul 23:1-9. German. doi: 10.1007/s00103-021-03386-x. Epub ahead of print. PMID: 34297162; PMCID: PMC8298983.

[4] Schmidt CO, Darms J, Shutsko A, Löbe M, Nagrani R, Seifert B, Lindstädt B, Golebiewski M, Koleva S, Bender T, Bauer CR, Sax U, Hu X, Lieser M, Junker V, Klopfenstein S, Zeleke A, Waltemath D, Pigeot I, Fluck J; NFDI4Health Task Force COVID-19. Facilitating Study and Item Level Browsing for Clinical and Epidemiological COVID-19 Studies. Stud Health Technol Inform. 2021 May 27;281:794798. doi: 10.3233/SHTI210284. PMID: 34042687.

[5] HL7. 2.16 FHIR Overview - Architects. [Internet]. 2019 [cited 2021 Aug 02]. Available from https://www.hl7.org/fhir/overview-arch.html

[6] Gruendner J, Wolf N, Tögel L, Haller F, Prokosch HU, Christoph J. Integrating Genomics and Clinical Data for Statistical Analysis by Using GEnome MINIng (GEMINI) and Fast Healthcare Interoperability Resources (FHIR): System Design and Implementation. J Med Internet Res. 2020 Oct 7;22(10):e19879. doi: 10.2196/19879. PMID: 33026356; PMCID: PMC7578821.

[7] Lee HA, Kung HH, Lee YJ, Chao JC, Udayasankaran JG, Fan HC, Ng KK, Chang YK, Kijsanayotin B, Marcelo AB, Hsu CY. Global Infectious Disease Surveillance and Case Tracking System for COVID19: Development Study. JMIR Med Inform. 2020 Dec 22;8(12):e20567. doi: 10.2196/20567. PMID: 33320826; PMCID: PMC7758088.

[8] Gulden C, Mate S, Prokosch HU, Kraus S. Investigating the Capabilities of FHIR Search for Clinical Trial Phenotyping. Stud Health Technol Inform. 2018;253:3-7. PMID: 30147028.

[9] HL7.org. HL7® FHIR ${ }^{\circledR}$ Release 4. [Internet]. 2019. [cited 2021 Aug 02]. Available from http://h17.org/fhir/R4/index.html

[10] Garza MY, Rutherford M, Myneni S, Fenton S, Walden A, Topaloglu U, Eisenstein E, Kumar KR, Zimmerman KO, Rocca M, Gordon GS, Hume S, Wang Z, Zozus M. Evaluating the Coverage of the HL7 ${ }^{\circledR}$ FHIR ${ }^{\circledR}$ Standard to Support eSource Data Exchange Implementations for use in Multi-Site Clinical Research Studies. AMIA Annu Symp Proc. 2021 Jan 25;2020:472-481. PMID: 33936420; PMCID: PMC8075534.

[11] NFDI4Health Task Force COVID-19 Metadata Schema (MDS) Mapping to FHIR - MDS-to-FHIR Mapping [Data Set] [Internet]. FAIRDOMHub; 2021. Available from: https://fairdomhub.org/data_files/4210?version=2

[12] HL7. HL7® FHIR $®$ Release 5 Draft Ballot -8.22 Resource ResearchStudy. [Internet]. 2021. [cited 2021 Aug 04]. Available from https://build.fhir.org/researchstudy.html

[13] SIMPLIFIER.NET. Medizininformatik Initiative. [Internet]. 2021. [cited 2021 Sep 16.] Available from https://simplifier.net/organization/koordinationsstellemii/ projects

[14] Rinaldi E, Thun S. From OpenEHR to FHIR and OMOP Data Model for Microbiology Findings. Stud Health Technol Inform. 2021 May 27;281:402-406. doi: 10.3233/SHTI210189. PMID: 34042774. 